\title{
Condensation in globally coupled populations of chaotic dynamical systems
}

\author{
D. H. Zanette* and A. S. Mikhailov \\ Fritz-Haber-Institut der Max-Planck-Gesellschaft, Faradayweg 4-6, 14195 Berlin, Germany
}

(Received 14 August 1997)

\begin{abstract}
The condensation transition, leading to complete mutual synchronization in large populations of globally coupled chaotic Rössler oscillators, is investigated. Statistical properties of this transition and the cluster structure of partially condensed states are analyzed. [S1063-651X(97)06012-1]

PACS number(s): 05.45.+b, 05.20. $-\mathrm{y}, 05.90 .+\mathrm{m}$
\end{abstract}

The appearance of condensates is an important property of quantum and classical systems. When a population of interacting Bose particles (such as, e.g., superfluid liquid ${ }^{4} \mathrm{He}$ ) is considered, a condensate is formed by a finite fraction of particles occupying the same quantum state [1]. Condensation of electron-hole pairs in metals is responsible for the phenomenon of superconductivity [2]. Condensation phenomena in classical systems are closely related to the effect of mutual synchronization of oscillations. In large populations of globally coupled limit-cycle oscillators, one or several groups of oscillators following exactly the same phase trajectory can spontaneously appear [3-7]. A similar behavior in populations of globally coupled logistic or circle maps has been found by Kaneko [8,9]. Mutual synchronization of several coupled chaotic oscillators has been studied (see, e.g., [10]). However, large populations of chaotic oscillators may also be globally coupled. This is typical, for instance, for surface chemical reactions where a great number of nanoscale dynamical microreactors on the catalytic surface are globally coupled through the gas phase [11]. The behavior of a large population of globally coupled chaotic Rössler oscillators has recently been investigated [12], but only weak synchronization leading to the appearance of statistical correlations has been discussed. In contrast to this, we analyze in this paper the statistical properties of a transition to the fully synchronous state that is found when a different form of global coupling is applied to the same system.

We find that the synchonization begins at low coupling intensities with the appearance of small coherent groups on the background of the rest of the population performing asynchronous oscillations. The elements belonging to such groups consititute a dynamical condensate. As the coupling strength is increased, the number of particles in the condensate grows and eventually the whole population becomes divided into several coherent clusters. Within each cluster, the elements follow the same chaotic dynamical trajectory. Under further increase of the global coupling, the number of coherent clusters gets reduced until full mutual synchronization is achieved. The aims of our study are to introduce appropriate order parameters for this condensation transition, to investigate distributions over the clusters, and to analyze the dependence of the condensation behavior on the system size.

\footnotetext{
*Permanent address: Consejo Nacional de Investigaciones Científicas y Técnicas, Centro Atómico Bariloche, 8400 Bariloche, Argentina.
}

We also consider how full mutual synchronization is influenced when random variations in the parameters of individual chaotic oscillators are introduced.

Not every form of global coupling between elements can lead to mutual synchronization. Synchronous chaotic oscillations are possible if "vector" coupling (with identical coupling coefficients for all oscillator variables) is employed [13]. Asymptotic synchronization conditions in the limit of high coupling intensities have also been constructed for the case when coupling involves only one of the oscillator variables [10]. However, only the linear stability of synchronous oscillations is proven in both of these cases and therefore even for a strong coupling the presence of other dynamical attractors, corresponding to persistent asynchronous oscillations, cannot be excluded [14].

In this paper a different form of global coupling that yields more robust synchronization is introduced. We take an arbitrary dynamical system with $m$ variables $\mathbf{w}(t)$ $=\left\{\mathbf{w}_{1}(t), \ldots, \mathbf{w}_{m}(t)\right\}$ whose evolution is determined by equations

$$
\dot{\mathbf{w}}=\mathbf{f}(\mathbf{w}),
$$

where $\mathbf{f}(\mathbf{w})$ are some known functions. A globally coupled population consisting of $N$ such dynamical systems ( $i=1,2, \ldots, N)$ is constructed by adding the coupling terms, so that the resulting evolution equations are

$$
\dot{\mathbf{w}}_{i}=\mathbf{f}\left(\mathbf{w}_{i}\right)+\varepsilon \hat{\mathbf{A}}\left(\overline{\mathbf{w}}-\mathbf{w}_{i}\right)+\varepsilon^{\prime}\left[\mathbf{f}(\overline{\mathbf{w}})-\mathbf{f}\left(\mathbf{w}_{i}\right)\right],
$$

where $\overline{\mathbf{w}}$ is the global average, $\overline{\mathbf{w}}(t)=N^{-1} \sum_{i=1}{ }^{N} \mathbf{w}_{i}(t)$, the positive parameters $\varepsilon$ and $\varepsilon^{\prime}$ specify the intensity of global coupling, and $\hat{\mathbf{A}}$ is a constant matrix. Note that the previously used forms of coupling correspond to a choice $\varepsilon^{\prime}=0$; the "vector"' coupling is obtained for $\hat{\mathbf{A}}=\mathbf{I}$ where $\mathbf{I}$ is the identity matrix.

When $\varepsilon^{\prime}=1$ Eq. (2) reduces to

$$
\dot{\mathbf{w}}_{i}=\varepsilon \hat{\mathbf{A}}\left(\overline{\mathbf{w}}-\mathbf{w}_{i}\right)+\mathbf{f}(\overline{\mathbf{w}}) .
$$

They describe linear motion of elements forming the population under action of a force that is collectively produced by all of them. The evolution of the deviations $\delta \mathbf{w}_{i}(t)$ $=\mathbf{w}_{i}(t)-\overline{\mathbf{w}}(t)$ is then determined by an exact linear set of equations $\delta \dot{\mathbf{w}}_{i}=-\varepsilon \hat{\mathbf{A}} \delta \mathbf{w}_{i}$. Therefore, these deviations exponentially decrease with time, and global stability of synchro- 
nous oscillations is thus guaranteed, if all real eigenvalues of the matrix $\hat{\mathbf{A}}$ and the real parts of all its complex eigenvalues are positive.

Since globally stable synchronous oscilations are present in the system (2) at $\varepsilon^{\prime}=1$, they may also be expected in a neighborhood of this point, i.e., for an interval of coupling intensities $\varepsilon_{0}<\varepsilon^{\prime}<1$. As shown below, this interval can be so wide that even weak coupling leads to synchronization.

We consider a population of chaotic Rössler oscillators. Each elementary oscillator is described now by three variables $\mathbf{w}(t)=\{x(t), y(t), z(t)\}$, and we have $f_{x}=-y-z$, $f_{y}=x+a y$, and $f_{z}=b-c z+x z$, where $a, b$, and $c$ are fixed parameters. The collective dynamics of a globally coupled population of $N$ identical Rössler oscillators $(i=1,2, \ldots, N)$ is governed by equations

$$
\begin{gathered}
\dot{x}_{i}=-y_{i}-z_{i}, \\
\dot{y}_{i}=x_{i}+a y_{i}+K\left(\bar{y}-y_{i}\right), \\
\dot{z}_{i}=b-c z_{i}+x_{i} z_{i}+K\left(\bar{x} \bar{z}-x_{i} z_{i}\right),
\end{gathered}
$$

where $\bar{x}(t), \bar{y}(t)$, and $\bar{z}(t)$ are global averages, such as $\bar{x}(t)=N^{-1} \sum_{i=1}^{N} x_{i}(t)$, and $K$ is the intensity of global coupling. This form of coupling is derived from Eq. (2) by taking $\varepsilon=\varepsilon^{\prime}=K$ and choosing a matrix $\hat{\mathbf{A}}$ whose elements are zero except for $A_{x y}=A_{x z}=-A_{y x}=1, \quad A_{z z}=c$, and $A_{y y}=1-a$. The system (4) reduces to a set of independent identical Rössler oscillators [15] for $K=0$.

The eigenvalues of the matrix $\hat{\mathbf{A}}$ are $\lambda_{1}=c$ and $\lambda_{2,3}=\frac{1}{2}(1-a) \pm \frac{1}{2} \sqrt{(1-a)^{2}-4}$. All three eigenvalues (or their real parts) are positive if $a<1$. Therefore, for $K=1$ all deviations from the global averages exponentially decrease with time and the states of all elements in the population asymptotically converge. We see that at $K=1$ the oscillator population (4) possesses a global attractor that corresponds to coherent motion of all elements of the system. This attractor coincides with the attractor of a single Rössler oscillator and is chaotic for the parameters $a=0.15, b=0.4$, and $c=8.5$ used in our simulations. Thus, the population (4) of globally coupled chaotic Rössler oscillators should undergo condensation as the coupling intensity $K$ is gradually increased towards $K=1$. To investigate the condensation phenomena, we have typically performed numerical simulations of a population of $N=1000$ such oscillators under varying intensity $K$ of global coupling.

The distances $d_{i j}=\left[\left(x_{i}-x_{j}\right)^{2}+\left(y_{i}-y_{j}\right)^{2}+\left(z_{i}-z_{j}\right)^{2}\right]^{1 / 2}$ between the states of all $N(N-1) / 2$ possible pairs of elements $(i, j)$ have been computed. When condensation occurs, the distances between some of the elements would decrease and asymptotically approach zero. Note, however, that in a computer simulation, the variables specifying the states of the elements are defined only up to a certain precision determined by the number of digits used to represent real numbers. Therefore, any two converging states become indistinguishable when all the digits coincide. Once this has occurred, the two trajectories remain identical at all subsequent times. Following [8], we assume that the distance between such two trajectories is zero.

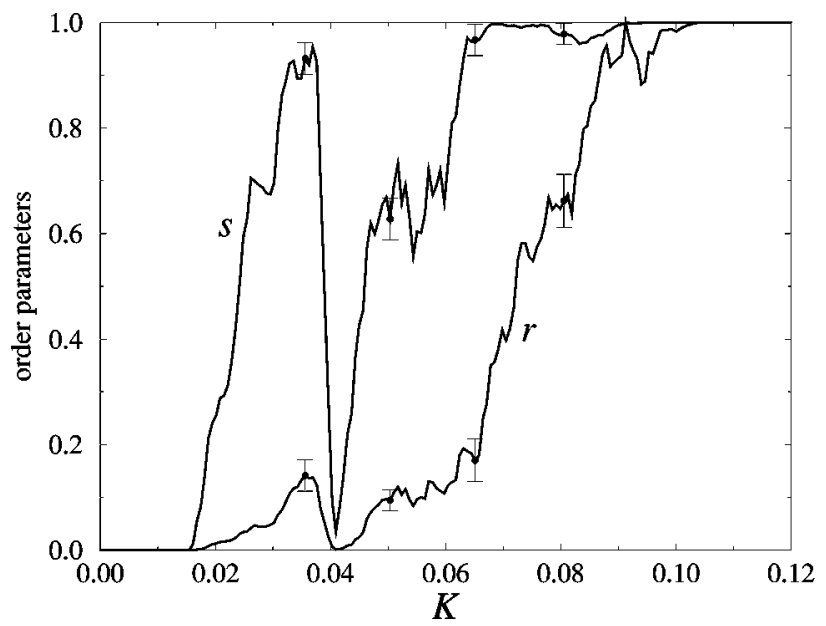

FIG. 1. Order parameters $r$ and $s$ of the condensation transition as a function of the coupling constant $K$. Averaging over 20 independent realizations with random initial conditions is performed; bars indicate statistical dispersion (mean square deviation) of data at several selected points.

To prepare the initial condition, the system is first allowed to evolve up to $t=100$ without global coupling $(K=0)$ so that its elements get uniformly distributed over the Rössler attractor. Global coupling is then introduced, time is reset to zero, and the evolution of the coupled population is started. We see that as time goes on the trajectories of some elements converge and become identical. These elements form the dynamical condensate. The transient is over when the number of elements in the condensate ceases to increase with time. In our simulations, the transients were always shorter than $t=1000$. This has been checked by following the evolution of the system for longer times at different values of the coupling intensity $K$, including the values of $K$ very close to the onset of the condensation transition.

To quantitatively characterize the condensation, two order parameters can be used. The first of them is given by the ratio $r$ of the number of pairs with zero distances to the total number of pairs. In the absence of a condensate, $r=0$. On the other hand, $r=1$ when complete mutual synchronization of the whole population takes place. The second parameter $s$ represents the fraction of the population belonging to coherent clusters. It is given by the relative number of elements that have at least one other element with the same state in the considered population. Therefore, $s$ can be viewed as characterizing the size of the condensate.

Figure 1 shows the dependence of the order parameters $r$ and $s$ on the global coupling intensity $K$ obtained by averaging over 20 realizations at $t=2000$. Condensation begins at $K_{c} \approx 0.017$ when the condensate first appears and a nonvanishing fraction of identical pairs becomes present. In the interval from $K_{c}$ to $K \approx 0.06$, nonmonotonous dependence of both order parameters on the coupling intensity is observed. At higher coupling intensities $K$, the condensate size and the fraction of identical pairs grow until full condensation $(r=1)$ is established for $K>K_{0}$, with $K_{0} \approx 0.1$. Under conditions of partial condensation, the condensate size and the number of identical pairs vary from one realization to another. Bars in Fig. 1 indicate statistical dispersion of the simulation data at a few selected values of the global cou- 


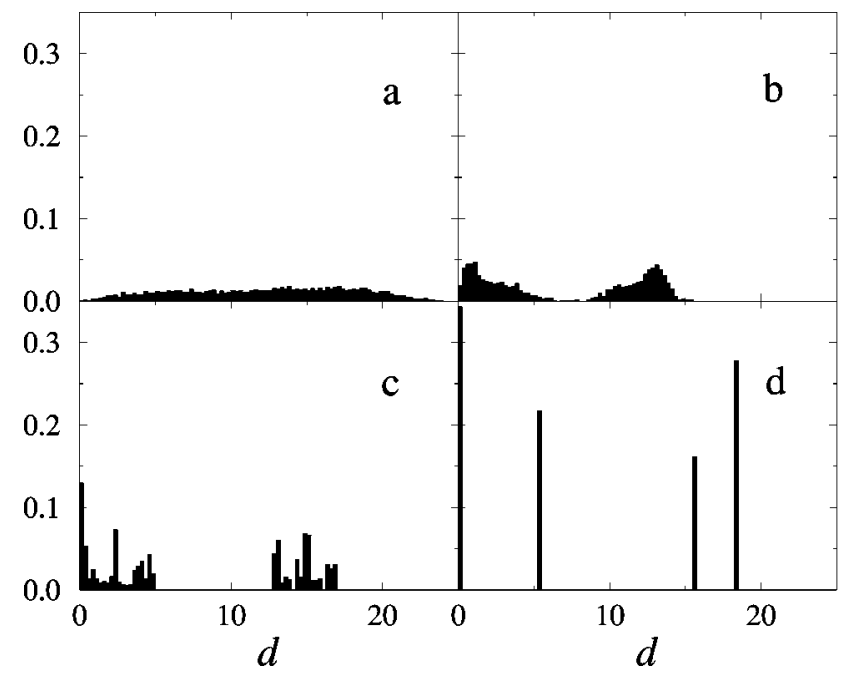

FIG. 2. Normalized histograms of the distribution over pair distances $d$ between elements in a population of $N=1000$ globally coupled Rössler oscillators at different stages of the condensation transition: (a) $K=0$; (b) $K=0.01$; (c) $K=0.02$; (d) $K=0.05$.

pling intensity. Note that the dispersion reveals a degeneracy in the asymptotic properties of this system in the partially condensed state.

The order parameters $r$ and $s$ characterize the overall degree of condensation, but are not sensitive to the condensate structure. To determine the detailed structure of the population, distributions over pair distances between the states of all elements at a fixed time moment $(t=2000)$ have been analyzed. Histograms of such distributions at four different coupling intensities $K$ are shown in Fig. 2. They are constructed by counting pairs with distances lying within subsequent intervals of width $\Delta d=0.25$. The number of pairs inside each interval, divided by the total number of pairs $N(N-1) / 2$, yields the height of the respective bar. In the absence of global coupling, elements are relatively uniformly distributed over the single-oscillator attractor and a smooth distribution over distances $d$ is thus observed [Fig. 2(a)]. When global coupling is introduced but remains below the critical point $K_{c}$ of the condensation transition, the distribution is modified [Fig. 2(b)]. Now, pairs with small distances have already appeared, though identical pairs are still absent (cf. Fig. 1). Slightly above the critical point, a strongly nonuniform distribution with narrow peaks is seen [Fig. 2(c)]. The distributions found at larger intensities of global coupling [e.g., Fig. 2(d)] are formed by several distinct lines. When complete condensation has taken place $(r=1)$, the histogram has only one line located at $d=0$ (not shown).

Distributions formed by several lines are characteristic for situations when the whole population breaks down into a number of coherent clusters. All elements in a cluster follow the same dynamical trajectory and, hence, distances between pairs of elements belonging to the same cluster are zero. When elements from two different clusters are chosen, their distances are all equal. If $M$ such clusters are present, the distribution over pair distances would consist of $M(M-1) / 2+1$ individual lines. For instance, four lines seen in Fig. 2(d) correspond to a condensate with three clusters. We have checked that the coherent motion of elements inside the clusters is not degenerated to simple periodic os-

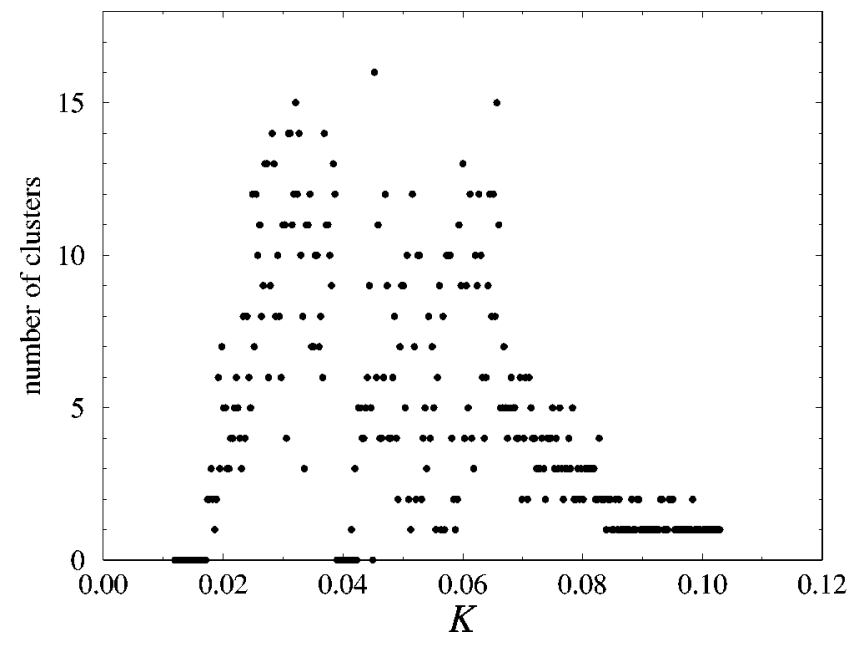

FIG. 3. Number of clusters counted at different values of the global coupling intensity $K$ separated by intervals of $\Delta K=3 \times 10^{-4}$ for a single simulation with random initial conditions at each point.

cillations and therefore this chaotic clustering is different from the behavior previously known for large populations of globally coupled limit-cycle oscillators (see [6]).

The cluster structure of the condensate as a function of the global coupling intensity $K$ has been analyzed. The dots in Fig. 3 show the numbers of clusters found at different coupling intensities separated by intervals $\Delta K=3 \times 10^{-4}$. For each value of $K$, a single simulation starting from random initial conditions has been performed. We see that the number of clusters is strongly fluctuating and may be largely different even for close values of the global coupling intensity. Even when the coupling intensity was fixed, the number of clusters varied from one simulation to another. Figure 4 shows the mean number of clusters as a function of the intensity $K$, averaged over 20 independent realizations. The bars show the statistical dispersion of the data.

The mean number of clusters can be estimated using the two order parameters $s$ and $r$. The total number of elements belonging to the condensate is $s N$. Assuming that the condensate consists of $M$ clusters of equal size, each cluster

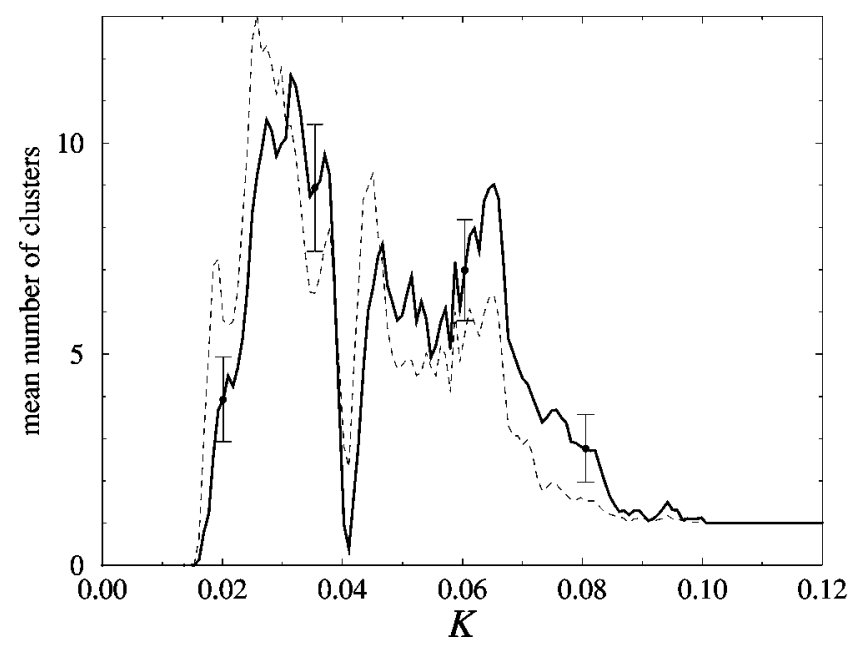

FIG. 4. Mean number of clusters (bold curve) and its estimate as $s^{2} / r$ (dash curve) as a function of $K$. Averaging over 20 independent realizations at each point; bars show the data dispersion. 


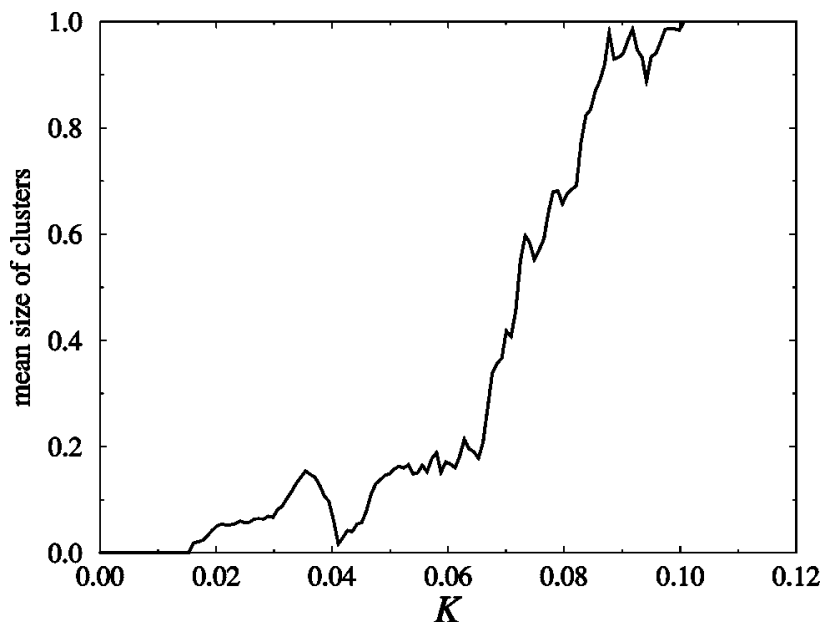

FIG. 5. The relative mean size of a cluster $n / N=r / s$ as a function of the coupling intensity $K$.

would contain $n=s N / M$ elements. The distances between the elements within any cluster are zero. Therefore, if the number $n$ of elements in each cluster is large, the total number of identical pairs in a single cluster would be approximately given by $n^{2} / 2$. Hence, the total number of identical pairs in the condensate is $M n^{2} / 2$. Since the total possible number of pairs is approximately $N^{2} / 2$, the order parameter $r$ is

$$
r=\frac{M n^{2}}{N^{2}}=\frac{s^{2}}{M} .
$$

Thus, the number of clusters can be estimated as $M=s^{2} / r$.

The dashed curve in Fig. 4 displays the mean number of clusters obtained using this estimate, which is valid if the clusters have equal sizes. It can be seen that generally it shows a reasonable agreement with the data obtained by direct counting of clusters. The deviations are due to the fact that the clusters have a distribution of sizes and some of them may be small. Note that the mean relative size of a cluster can be estimated as $n / N \approx r / s$. Figure 5 shows the dependence of the mean cluster size, obtained using this estimate, on the coupling intensity.

Thus, the condensation transition leading to complete mutual synchronization of the whole population can be described as follows. The condensate appearing above the transition point includes only a small fraction of elements, so that the order parameter $s$ is small in this region. The condensed elements are distributed over a few small clusters that represent nuclei of the condensed state. The rest of the population has asynchronous dynamics, though it is already essentially influenced by global coupling between the elements, as evidenced by the histogram shown in Fig. 2(c). When the coupling intensity is increased, the number of clusters rapidly grows and reaches a maximum of about 10 at $K \approx 0.03$. At the same time, the mean size of clusters slowly increases up to a few tens. After that a sudden desynchronization at $K \approx 0.04$ is observed. The number of clusters drops down and, in some realizations, synchronization is even completely lost (Fig. 3). The few persisting clusters are small in this region. Under further increase of the global coupling intensity, synchronization is gained back. Now, both the number of coherent clusters and their mean size steadily grow and approach intermediate saturation in the interval from about $K \approx 0.045$ to $K \approx 0.065$. Here the typical size of a cluster is about 150 . At the end of this interval the clusters forming the condensate already include almost all population members $(s \approx 1)$. At larger coupling intensities, redistribution of elements over a decreasing number of clusters is observed. Eventually, at $K \approx 0.1$ only one cluster is typically found (although occasionally two clusters may also appear close to this point). This means that full mutual synchronization has been achieved.

Complete condensation $(r=s=1)$ has been found within a wide interval of the global coupling intensity. Starting in this region with random initial conditions for all elements, we see that the elements soon form a compact cloud that rapidly shrinks with time. The characteristic radius $R(t)$ of this cloud can be defined by

$$
R^{2}(t)=\frac{1}{N} \sum_{i=1}^{N}\left[\delta x_{i}^{2}(t)+\delta y_{i}^{2}(t)+\delta z_{i}^{2}(t)\right]
$$

where $\delta x_{i}, \delta y_{i}$, and $\delta z_{i}$ are the deviations from the respective global averages. The radius $R$ decreases with time, as the trajectories of all elements asymptotically converge to the same orbit (in our simulations the convergence was followed until the trajectories of all elements became identical up to the computer precision of about $10^{-6}$ ). An important quantitative property of this regime is the characteristic time that the system needs to reach the condensed state; the inverse of this time represents the condensation exponent (closely related to the transverse Lyapunov exponents discussed in Ref. [10]):

$$
\gamma=-\lim _{t \rightarrow \infty} \frac{1}{t} \ln \frac{R(t)}{R(0)} .
$$

The simulations show that $\gamma$ starts to grow from zero at $K=K_{0}$, has a maximum at $K \approx 0.65$, and then slowly decreases, approaching the value $\gamma \approx 0.38$ at $K=1$.

The robustness of the complete condensation suggests that it might persist in some form even in heterogeneous populations. To test this, we have carried out simulations where the elements forming the population were not identical. Heterogeneity was introduced by replacing the constant parameter $c$ in Eqs. (4) by random numbers $c_{i}$ that were uniformly distributed inside the segment $\left[c-\sigma_{c}, c+\sigma_{c}\right]$. Alternatively, the same procedure was applied to the parameter $a$. Starting from random initial conditions, a compact cloud of elements was again formed in the heterogeneous case. However, the cloud did not shrink until it transformed into a single point. Instead, after an initial decrese, its radius $R(t)$ fluctuated around a certain mean value. Figure 6 shows the mean radius $\langle R\rangle$ as a function of dispersions $\sigma_{c}$ or $\sigma_{a}$ for $K=0.2$. We see that in a wide interval of heterogeneities the mean radius of the cloud is approximately linear proportional to the dispersion. Moreover, even for relatively large dispersions this radius is significantly smaller that the characteristic attractor diameter $D$, representing the maximal possible pair distance at $K=0$ [i.e., $D \approx 25$, cf. Fig. 2(a)]. Therefore, even though the states of all elements are no longer identical (i.e., the complete condensation is destroyed), their variations re- 


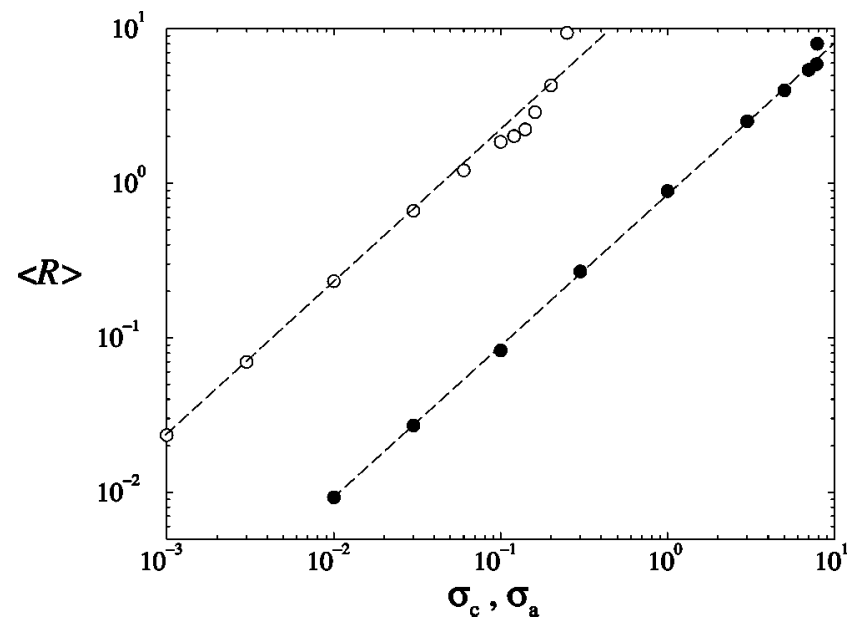

FIG. 6. Radius $\langle R\rangle$ of the cloud formed by a heterogeneous population as function of dispersions $\sigma_{a}$ (empty circles) and $\sigma_{c}$ (filled circles) at $K=0.2$.

main small. The elements form a little cloud whose center moves along a definite trajectory.

This behavior can be understood by analyzing the effect of weak heterogeneities at the maximal strength of global coupling $K=1$. If the parameter $c$ is replaced by $c_{i}=c+\Delta c_{i}$ with random variations $\Delta c_{i}$, the population dynamics is described by

$$
\begin{gathered}
\dot{x}_{i}=-y_{i}-z_{i}, \\
\dot{y}_{i}=x_{i}+(a-1) y_{i}+\bar{y}, \\
\dot{z}_{i}=b-c_{i} z_{i}+\bar{x} \bar{z} .
\end{gathered}
$$

Hence, motion of each element is governed by linear equations of motion in the presence of global driving forces $\bar{y}$ and $\bar{x} \bar{z}$, which are the same for all elements. Therefore, deviations $\delta z_{i}$ from the global average obey the equation

$$
\delta \bar{z}_{i}=-c \delta z_{i}-\Delta c_{i} z-\Delta c_{i} \delta z_{i}+\overline{\Delta c_{i} \delta z_{i}} \text {. }
$$

If heterogeneities are weak, i.e., $\Delta c_{i}=\theta \zeta_{i}$ where $\theta$ is a small parameter, the last two terms in this equation have order $\theta^{2}$ and can be neglected. Integrating the resulting linear equation, we obtain

$$
\delta z_{i}(t)=\theta \zeta_{i} \int_{0}^{\infty} \exp (-c \tau) \bar{z}(t-\tau) d \tau .
$$

Considering the behavior of other deviations $\delta x_{i}$ and $\delta y_{i}$, one can similarly show that they are also proportional to $\Delta c_{i}=\theta \zeta_{i}$. This means that the radius $\langle R\rangle$ of the population cloud depends as $\langle R\rangle \sim \theta$ on the heterogeneity strength. Note also that in this case all deviations are scaling identical, i.e., for example, $\delta z_{i}(t)=\theta \zeta_{i} Q(t)$ where the function $Q(t)$ is the same for any element $i$. The same results are obtained when weak random variations of the parameter $a$ are considered.

The simulations have revealed that the linear dependence of the mean radius $\langle R\rangle$ on the dispersions $\sigma_{c}$ and $\sigma_{a}$, that

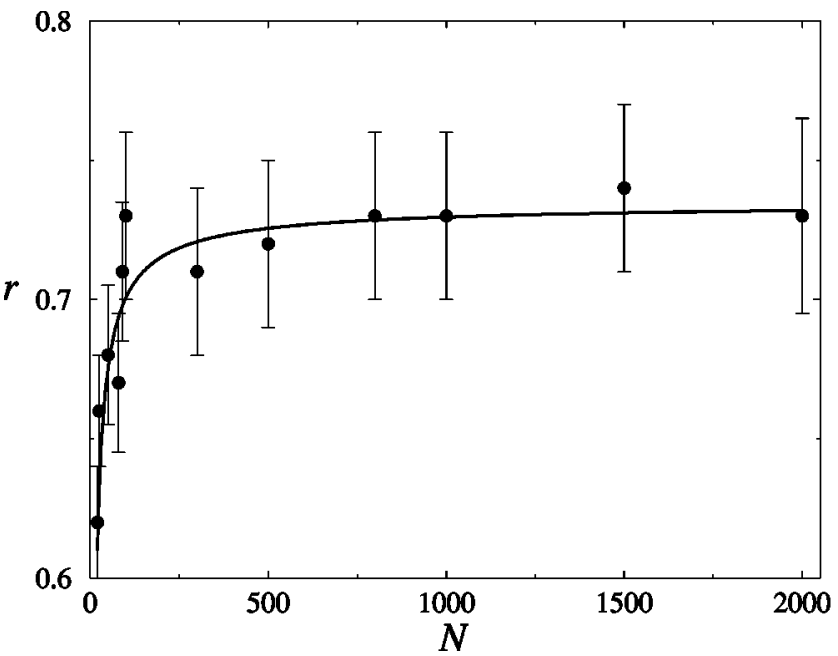

FIG. 7. Dependence of the order parameter $r$ on the population size $N$ at a fixed intensity of global coupling $K=0.08$. Averaging over 20 independent realizations; bars indicate statistical dispersion of data.

has been analytically shown above only at $K=1$, persists in a wide range of coupling intensities. Moreover, this dependence is found even for relatively large dispersions, where the parameter $\theta$ would not be small. This behavior is apparently a consequence of a strong compression provided in the entire interval of complete condensation by global coupling.

Our analysis has been performed for a population comprising 1000 chaotic Rössler oscillators. The question may arise whether the statistical behavior found for a population of this size is already characteristic for a limit of very large systems. To answer it we have done a series of simulations with varying population sizes. Figure 7 shows the typical dependence of the order parameter $r$ of the condensation transition on the total population size $N$, where each point is obtained by averaging over 20 independent realizations and the intensity of global coupling is kept constant $(K=0.08)$. We see that a strong size dependence is characteristic for relatively small populations $(N<500)$. Starting from a population size of about $N=1000$, the dependence displays saturation, with the remaining small variations lying within the statistical dispersion range. Hence, the results of our study of a population with 1000 oscillators might already be representative for the asymptotic behavior in the infinite-size limit $N \rightarrow \infty$. The condensation transition has been studied above for a particular kind of chaotic oscillator and for a certain form of global coupling. We have, however, checked that similar results are also obtained when a different ("vector") form of global coupling is used. This suggests that observed statistical properties may be typical for various large populations of globally coupled chaotic oscillators undergoing a condensation transition.

Financial support from Fundación Antorchas, Argentina, and from Alexander von Humboldt-Stiftung, Germany, is gratefully acknowledged. D.H.Z. wishes to thank the Fritz Haber Institute for hospitality during his stay in Berlin. 
[1] N.N. Bogoliubov, Lectures on Quantum Statistics, Vol. I (Gordon and Breach, New York, 1967).

[2] H. Umezawa, H. Matsumoto, and M. Tachiki, Thermo-Field Dynamics and Condensed States (North-Holland, Amsterdam, 1982).

[3] Y. Kuramoto, Chemical Oscillations, Waves and Turbulence (Springer, Berlin, 1984).

[4] N. Nakagawa and Y. Kuramoto, Physica D 75, 74 (1994).

[5] H. Sakaguchi and Y. Kuramoto, Prog. Theor. Phys. 76, 576 (1986).

[6] V. Hakim and W.-J. Rappel, Phys. Rev. A 46, R7347 (1992).

[7] D. Golomb, D. Hansel, B. Shraiman, and H. Sompolinsky, Phys. Rev. A 45, 3516 (1992).

[8] K. Kaneko, Physica D 23, 436 (1986); 37, 60 (1989).

[9] K. Kaneko, Physica D 54, 5 (1991).
[10] J. F. Heagy, T. L. Carrol, and L. M. Pecora, Phys. Rev. E 50, 1874 (1994).

[11] N. Khrustova, G. Veser, A. Mikhailov, and R. Imbihl, Phys. Rev. Lett. 75, 3564 (1995).

[12] A.S. Pikovsky, M.G. Rosenblum, and J. Kurths, Europhys. Lett. 34, 165 (1996).

[13] H. Fujisaka and T. Yamada, Prog. Theor. Phys. 69, 32 (1983).

[14] In the complex Ginzburg-Landau equation describing a population of locally coupled limit-cycle oscillators, both linearly stable synchronous uniform oscillatons and complex spatiotemporal patterns, such as spiral waves or turbulence, can often be found depending on the initial conditions at the same system parameters; see: B. I. Shraiman, A. Pumir, W. von Saarlos, P. C. Hohenberg, H. Chaté, and M. Holen, Physica D 57, 241 (1995).

[15] O. E. Rössler, Phys. Lett. A 57, 97 (1976). 